\title{
CURRENT THERAPEUTIC RESEARCH
}

AnNual Index, Volume $7 \mathrm{I}, 20 \mathrm{IO}$

\section{Author Index}

Abdulamir AS, 60

Abubaker F, 60

Ajili F, 398

Aksu R, 111, 289

Alakus MF, 173

Altun G, 118

Ark N, 105

Aulakh BS, 273

Aydin M, 369

Barbouche M-R, 398

Beheshtiha SHS, 322

Ben Ali M, 398

Ben Mustapha I, 398

Bicer C, 111, 289

Bolla GB, 309

Boubaker S, 398

Boyaci A, 111, 289

Brasca F, 309

Caimi B, 309

Caparlar CO, 408

Carugo S, 309

Cekmen N, 408

Chebil M, 398

Chen H, 384

Cherif M, 398

Cui F, 384

D'Andrea C, 50

Demirkiran H, 141

Derouiche A, 398

Diao X, 30

Dixit VK, 331

Dogan Z, 141

Doğru K, 111

Eaves AM, 384

Ekerbicer H, 141
Erkasap N, 186

Erkasap S, 186

Eskin MB, 408

Famiani R, 309

Fan T, 30

Fu J-J, 30

Fujii Y, 154

Gou J-F, 211

Guler G, 289

Gunduz M, 105

Gupta K, 273

Hafidh RR, 60

Hatipoglu AR, 118

İhtiyar E, 186

Ishigami S, 162

Jeon Y, 78, 298

Jia J-Y, 260

Jouyban A, 322

Kaçmaz M, 111

Kang JG, 93

Kara IH, 173

Kaur K, 273

Kaushal S, 273

Keklikci U, 173

Keppel Hesselink JM, 416 (letter)

Kim H, 78

Kim S, 298

$\operatorname{Kim} \mathrm{TH}, 93$

Kim YC, 93

Kitazono M, 162

Köken T, 186

Kopsky DJ, 416 (letter)
Kosar A, 105

Kurtaran H, 105

Kwak K-H, 78

Lee CJ, 93

Lee PB, 93

Lee SC, 93

Lee $\mathrm{SH}, 93$

Leucuta SE, 360

Li D-C, 239

Li M, 129

Li S-J, 260

Li S-W, 199

Li W-X, 211

Li X-C, 260

Liu F, 1

Liu G-J, 30

Liu G-Y, 260

Liu X-M, 30

Liu Y, 260

Liu Y-M, 260

Lu Y-L, 260

Madenoğlu H, 111

Magrini F, 309

Mannai M, 398

Mao B, 30

Miao J, 384

Michishita I, 252

Mizuguchi I, 252

Muntean D, 360

Nacar A, 369

Nahata A, 331

Nahm FS, 93

Nandecha C, 331

Natsugoe S, 162

Neag M, 360

Nozue T, 252 
Oksuz H, 141

Oktar S, 369

Öner S, 186

Ozboduroglu AA, 105

Özdemir R, 186

Ozhan MO, 408

Ozkan OV, 369

Ozturk OH, 369

Phetdee K, 345

Popa A, 360

Qiu M, 1

Rashidi S, 322

Rezvanjoo B, 322

Rossetti G, 309

Sacks H, 50

Sakalar YB, 173

Samini M, 322

Senoglu N, 141

Seo J, 298

Sert H, 105

Sezer A, 118

Sheng W, 384

Shin BS, 93
Shinchi H, 162

Sim WS, 93

Simon S, 50

Sogut S, 369

Sut N, 118

Suzer MA, 408

Tengamnuay I, 345

Tian J-H, 211

Tosun M, 186

Tosun Z, 289

Tuntijarukorn P, 345

Uemura A, 154

Ueno S, 162

Ugur KS, 105

Unlu K, 173

Usta U, 118

Viyoch J, 345

Vlase L, 360

Walson JL, 91

Wang H, 384

Wang J, 129

Wang J, 384

Wang Y, 199
Wang Y-P, 260

Waranuch N, 345

Wheeler WJ, 50

Xu Z, 129

Xue T, 199

Yaba G, 111

Yan X, 211

Yang H-M, 30

Yang L, 211

Yaşar NF, 186

Yetim I, 369

Yildiz H, 141

Yildiz K, 111

Yilmaz N, 369

Yu C, 260

Yu X, 384

Zhai S-D, 1

Zhang W-B, 30

Zhang Z-X, 239

Zhao Q, 384

Zhong N, 384

Zhong Y-Q, 30

Zhou J, 239 
Subject Index 2010

Acetaminophen, antinociceptive effects, 111

Acetylsalicylic acid, gastric damage, 369 trans-4-(Aminomethyl)

cyclohexanecarboxylic acid, and potassium azeloyl diglycinate and niacinamide topical emulsion combination, melasma, Thailand, 345

(S)-Amlodipine, 1

Analgesic effects, ketamine infusion, 93

Anesthetics

ketamine, 93, 289

lidocaine, 289

ropivacaine, 298

Angiotensin-receptor blocker, valsartan, 309

Antiemetics

dexamethasone, 78

ramosetron, 78

Antifungal, griseofulvin, 384

Antimuscarinic, fesoterodine, 273

Antinociception, effects of naloxone and flumazenil with acetaminophen, 111

Apoptosis, K562 leukemia cells, 384

Bacille Calmette-Guérin, genetic polymorphisms, bladder cancer, 398

Benincasa hispida, 331

Benzodiazepines

flumazenil, 111

midazolam, 141, 298

Bladder cancer, Bacille Calmette-Guérin, genetic polymorphisms, 398

Bromocriptine, clomiphene, infertility, 199

Calcium channel blockers

$(S)$-amlodipine, 1 racemic amlodipine, 1

Cancer

bladder, Bacille Calmette-Guérin, genetic polymorphisms, 398 leukemia $K 562$ cells, griseofulvin, 384

pancreatic

lorglumide, cell line $\mathrm{Mia} \mathrm{PaCa}-2$ through cholecystokinincholecystokinin-1 receptor pathways, 239

sodium butyrate, 162

Cardiovascular damage, valsartan, 309

Carisoprodol, 50

Caspases, 3- and 9-dependent pathways, K562 leukemia cells, 384

Caustic sclerosing cholangitis, melatonin, 118

Central serous chorioretinopathy, verteporfin and photodynamic therapy, 173

Charles R. Ream, MD, Award for Excellence, 91

Chinese herbs, effects of dentifrice and, on volatile sulfur compounds, 129

Chinese patients depside salts from Salvia miltiorrbiza, pharmacokinetics of (special populations), 260

traditional Chinese medicine compound formulations, reporting quality, scientific rigor, ethics, 30

Cholecystokinin antagonist, lorglumide, 239

Cholecystokinin-cholescystokinin-1

receptor pathway, 239

Cholesterol absorption inhibitor, ezetimibe, 252

Clomiphene, bromocriptine and, infertility, 199

CNS depressants carisoprodol, 50 meprobamate, 50

Cough, in patients undergoing surgery, 289

Cysteamine-induced duodenal ulcer, 322 
Dentifrice, effects of Chinese herbs and, on volatile sulfur compounds, 129

Depside salts, 260

Dexamethasone, and ramosetron, women undergoing thyroidectomy, Korea, 78

Dexmedetomidine, 141

Diabetes ezetimibe, glucose metabolism, type 2,252

glucagon-like peptide- 1 receptor agonists versus insulin glargine, type 2, 211

Diaphragmatic contraction, dibutyryl cyclic adenosine monophosphate, 154

Dibutyryl cyclic adenosine monophosphate, diaphragmatic contraction, 154

Diuretic, Benincasa bispida, 331

Dopamine receptor agonist, bromocriptine, 199

Doxycycline, 186

Epidural analgesia, 298

Epistaxis, 105

Ethics, traditional Chinese medicine compound formulations, 1

Ezetimibe, 252

Fentanyl, 289, 298

Fesoterodine, overactive bladder, 273

Flumazenil, naloxone and, 111

Fluoxetine, omeprazole, 360

Galactorrhea, 199

Gastrectomy, antiemetic effects of midazolam and fentanyl-ropivacaine combination, 298

Gastric damage, $\beta$-glucans, 369

Genetic polymorphisms, 398

Glucagon-like peptide-1 receptor agonists, type 2 diabetes mellitus, 211

$\beta$-Glucans, gastric damage, 369

Glucose metabolism, 252

Griseofulvin, 384
Halitosis, 129

Hemostatic agents, 105

Hypertension

abdominal compartment syndrome, 186

amlodipine, racemic versus $(S)$-, 1

valsartan, 309

Infertility, bromocriptine and clomiphene, 199

Insulin glargine, type 2 diabetes mellitus, 211

Intestinal ischemia reperfusion injury, doxycycline, 186

Ketamine analgesic effects, neuropathic pain, Korea, 93 lidocaine or, fentanyl-induced cough, 289

Korean patients dexamethasone, and ramosetron, women undergoing thyroidectomy, 78 neuropathic pain and ketamine infusion, 93

Lidocaine, or ketamine, fentanyl-induced cough, 289

Melasma, 345

Melatonin effects, caustic sclerosing cholangitis, 118 vitamin $\mathrm{C}$ and, cysteamine-induced duodenal ulcer, 322

Meprobamate, 50

Midazolam, 141, 298

Muscle relaxant, carisoprodol, 50

Naloxone, flumazenil and, 111

Neuropathic pain, 93

Niacinamide topical emulsion, and potassium azeloyl diglycinate and trans-4-(aminomethyl) 
cyclohexanecarboxylic acid combination, melasma, Thailand, 345

Noninvasive mechanical ventilation, sedation and, 141

Nonopioid analgesics acetaminophen, 111 acetylsalicylic acid, 369

Omeprazole, fluoxetine and, 360

Opioid analgesic, fentanyl, 289, 298

Opioid antagonist, naloxone, 111

Overactive bladder, fesoterodine, 273

Ovulatory stimulant, clomiphene, 199

Pancreatic cancer lorglumide on cancer cell line Mia PaCa-2 through cholecystokinin-cholescystokinin-1 receptor pathways, 239 sodium butyrate on cancer cells, 162

Pharmacokinetics depside salts from Salvia miltiorrbiza, in Chinese patients, 260

fluoxetine, and omeprazole, 360

Potassium azeloyl diglycinate, and niacinamide topical emulsion and trans-4-(aminomethyl)

cyclohexanecarboxylic acid combination, melasma, Thailand, 345

Prostatic hypertrophy, testosteroneinduced, Benincasa hispida fruits and, 331

53-kDa Protein expression and sensitivity, 162

Proton pump inhibitors, omeprazole, 360

Psychotropics, meprobamate, 50

Racemic amlodipine, 1

Ramosetron, and dexamethasone, in women undergoing thyroidectomy, Korea, 78

Reporting quality, and traditional Chinese medicine compound formulations, 1

Ropivacaine, 298
Salvia miltiorrbiza, 260

Scientific rigor, and traditional Chinese medicine compound formulations, 1

Sedation, noninvasive mechanical ventilation and, 141

Selective serotonin reuptake inhibitor, fluoxetine, 360

Shoulder arthroscopy, 408

Sodium butyrate, 162

Special populations

Chinese patients depside salts from Salvia miltiorrbiza, pharmacokinetics, 260

traditional Chinese medicine compound formulations, reporting quality, scientific rigor, ethics, 30

Korean patients

dexamethasone, and ramosetron, in women undergoing thyroidectomy, 78

neuropathic pain and ketamine infusion, 93

Thai patients

potassium azeloyl diglycinate, and niacinamide topical emulsion and trans-4-(aminomethyl) cyclohexanecarboxylic acid combination, melasma, 345

women dexamethasone, and ramosetron, thyroidectomy, Korea, 78

Thai patients

potassium azeloyl diglycinate, and niacinamide topical emulsion and trans-4-(aminomethyl) cyclohexanecarboxylic acid combination, melasma, 345

Thyroidectomy, in Korean women, 78 Tracheal compression, 408

Traditional Chinese medicine compound formulations, reporting quality, scientific rigor, ethics, 30 
Ulcer, cysteamine-induced duodenal, 322

Vaccines

Bacille Calmette-Guérin, bladder cancer, 398

23-Valent pneumococcal polysaccharide, zwitterionized versus nonzwitterionized, 60
23-Valent pneumococcal polysaccharide, zwitterionized versus nonzwitterionized, 60

Valsartan, 309

Verteporfin, 173

Vitamin C, 322

Volatile sulfur compounds, 129

Women, dexamethasone, and ramosetron, thyroidectomy, Korea, 78 